ISBN $\quad 82-553-0478-9$

Mathematics

February 3

No. 3

1982

\title{
DEGENERATIONS OF CORPLETE
}

TNISTED CUBICS

by

Ragni Piene

Inst. of Rath. University of oslo

PREPRINT SERIES - Matematisk institutt, Universitetet i oslo 
1. Introduction

Let $C \in \mathbb{P}^{3}$ be a twisted cubic curve. Denote by $\Gamma$ C (rass $(1,3)$ its tangent curve (curve of tangent lines) and by $C^{*}<\mathscr{P}^{3}$ its dual curve (curve of osculating planes). The curve $\Gamma$ is rational normal. of degree 4 , while $C^{*}$ is again a twisted cubic. The triple $\left(C, \Gamma, C^{*}\right)$ is called a (non derenerate) complete twisted cubic. By a degeneration of it we mean a triple $\left(\overrightarrow{\mathrm{C}}, \vec{\Gamma}, \overrightarrow{\mathrm{C}}^{*}\right)$, where $\overrightarrow{\mathrm{C}}$ (resp. $\vec{\Gamma}$, resp. $\left.\bar{C}^{*}\right)$ is a flat specialization of $C$ (resp. $\Gamma$, resp. $C^{*}$ ). Thus we work ith lilbert schemes rather than chow scherles: let $H$ denote the irreducible component of $H_{i l b}{ }^{3 n+1}\left(m^{3}\right)$ containine, the twisted cubjos, II the corresponding component of $\mathrm{HjIh}^{3 \mathrm{n}+1}\left(\mathbb{P}^{3}\right)$, and $\mathrm{G}$ the component of Hilb ${ }^{4 m+1}(\operatorname{Grass}(1,3))$ contafning the tangent curves of twisted cubics. The space of complete twisted cubics is the closure $\because \mathrm{H} \times \mathrm{G} \times \mathrm{II}$ of the set of non degenerate complete twister cuhics.

In this paper we show how to obtain Schubert's 11 first order desenerations ( $[s], \mathrm{pp} .164 \times 166)$ of complete twisted cubjos, viewed as elements of $\mathrm{H} \times \mathrm{G} \times$ II., "via projections", $i . e .$, by constructing 1 dimensional families of curves on various linds of cones. In particular, we describe the ideals of the derrenerated curves. A similar study was done by Alguneid [A], who viewed the desenerations as cycles (rather than flat specializations), and who gave equations for the corrolexes of lines associated to the degenerated cycles by usine the thoory of complete collineations.

An ultimate cokl in the study of degenerations of complete twisted cubics, is of course to verify schubert's results in the enurerative theory of twisted cubics. As long as one, as schubert does, postricts oneself to only jupose conditions that involve points, tanrents, and osculating planes (and not secants, chords, osculatins linos, ...), the space $m$ is a compactification of the space of twisted cubics that contains enough information. In other words, one would like to describe the chow ring of $\mathrm{T}$ in tems of cycles corresponding to degenerate complete 
twisted cubics, and in terns of cycles representing the various schubert conditions. One approach rould be to study the chow ring of $I$ and the blow-up map $m \rightarrow H$ In a joint work with ilohael Schlessinger we prove that the 12-rimensional scheme $\mathrm{Il}$ is in fact srooth, and, roreover, that Il intersects the other (15-dinensional) component $\mathrm{Ii}^{\prime}$ of $\mathrm{Hilb} \mathrm{n}^{3 \mathrm{t}}\left(\mathrm{P}^{3}\right)$ transversaly along an 11 - dimensional locus (II' contains plane cubio curves union a point in $\mathbb{P}^{3}$, and $H \cap H^{\prime}$ consists of plare cubics with sn enbedded point) misis result, together with further investifations of the map $\mathrm{T} \rightarrow \mathrm{H}$, will be the subject of a forthooning paper.

Acknowledgments. This rork was begun while the author was a visitor of the Department of Tathematics, Unjversidade Federal de Penambuco, tu March/April 1981. Nany thanks are due to Prof. I. Vainsencher for conversations that started the work, and to ClPa for its financial support.

\section{Defenerations via projections}

Since all twisted cubics are projectively equivalent, we shall $\mathrm{fix}$ one, $\mathrm{C} C \mathbb{P}^{3}=\mathbb{P}_{\mathrm{K}}^{3}$ ( $\mathrm{k}$ algebraically closed field of characteristic 0 ), given by the ideal

$$
I=\left(X_{0} X_{2}-x_{1}^{2}, X_{1} X_{3}-x_{2}^{2}, X_{0} X_{3}-x_{1} x_{2}\right)
$$

Ilence $C$ has a parameter fom

$$
x_{0}=u^{3}, x_{1}=u^{2} v, x_{2}=u v^{2}, x_{3}=v^{3} \text {. }
$$

The tangent curve $\Gamma$ of $C$, viewed as a curve in $\mathbb{T}^{5}$ via the Plicker embeding of crass $(1,3)$, has a parameter form $\left(t=\frac{v}{u}\right)$ given by the 2manors of

$$
\left(\begin{array}{llll}
1 & t & t^{2} & t^{3} \\
0 & 1 & 2 t & 3 t^{2}
\end{array}\right)^{\prime}
$$

hence by

$$
\begin{aligned}
& Y_{0}=v^{4}, Y_{1}=2 u v^{3}, Y_{2}=v^{2} v^{2}, \\
& I_{3}=3 u^{2} v^{2}, I_{4}=2 u^{3} v, Y_{5}=u^{4} .
\end{aligned}
$$


The ideal of $\Gamma$ in $\mathbb{P}^{5}$ is

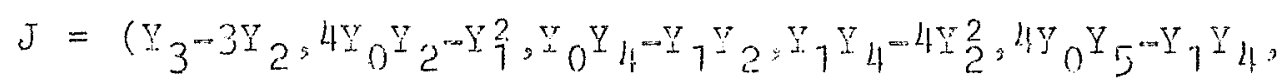
$\left.Y_{7} Y_{5}-Y_{2} Y_{4}, 4 Y_{2} Y_{5}{ }^{-x} Y_{i}\right)$.

The oual curvo ${ }^{*} \mathrm{c} \mathrm{i}^{3}$ hes a pamaneter form given by the 3-minors of

$$
\left(\begin{array}{llll}
1 & t & t^{2} & t^{3} \\
0 & 1 & 2 t & 3 t^{2} \\
0 & 0 & 1 & 3 t
\end{array}\right)
$$

hence by

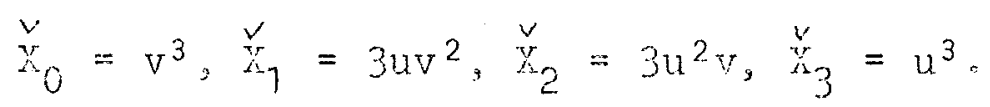

Since $\Gamma$ is also equal to the tangent curve of $C^{*}$. (under the canonical isomopphisn Crass(linos in $\mathbb{P}^{3}$ ) (rrass(lines in $\left.{ }^{3}\right)$ ) and $C$ is the dual curve of $C^{*}$ (see e.g० $[\mathrm{P}], \delta 5)$, any type of degeneration $\left(\overline{\mathrm{C}}, \bar{\Gamma}, \mathrm{C}^{*}\right)$ (jives another type - called the dual deseneration - by reading the triple backwards.

Let $A C \mathbb{P}^{3}$ be a linear space, and choose a complement $B C \mathbb{P}^{3}$ of $A$. By projectins $C$ onto $B$ from the vertex $A$ we obtain a deseneration of $C$ : we construct a farily $\left\{\mathrm{C}_{\mathrm{a}}\right\}$ of twisted cubics, contained in the cone of the above projection, over spec $k[a]-\{0\}$. mhis fanily has a unique extension to a flat family over spec k[a], and the "Iimit curve" $C_{0}$ is thus a flat specialization of $\mathrm{C}=\mathrm{C}_{1}$ (see also [II], 0.259 , for the case $A=a$ point). lote that interchanging the roles of $A$ and $B$ gives a linit curve equal to the curve $C_{\infty}$ obtained in the sinilar way by letting $a \rightarrow \infty$, and $C_{\infty}$ has the dual degeneration type of $\mathrm{C}_{0}$. The tyre of degeneration obtajned depends of course on the dimension and position of $A$ and $B$ wor.t. C.

To find senerators for the ideals of the desenerated curves, for chosen $A$ and $B$, we start by miting down a parameter forn of $\mathrm{C}_{\mathrm{a}}$, $\mathrm{a} \neq 0$. (It is often convenient to introduce new coordinates at this point.) Then we deterrine enough cenerators for the ideal I a of Cas so that they specialize $(a=0)$ to senerators for the ideal $I_{0}$ of $\mathrm{C}_{0}$. (Whenever $\mathrm{C}_{0}$ accuires an embedded point, it turns out that a cubic generator is needed in addition to 
the (three standard) quadratic ones.)

whe rarameter form of $\mathrm{C}_{a} a * 0$, sives a paremeter form of $\Gamma_{A}$, its tangent curve. As above we find generators for the ideal I of $\Gamma_{a}$ that specialize to Generators for Jo.

Sirilarly, one could work out the jieal of $C_{0}^{*}$. llowever, by a duality argunent it is clear that ${ }^{\circ} 0$ will. have the degeneration type obtained (Iron C) by inter.. changing the roles of $A$ and $B$. That is, $C_{0}^{*}$ will be of the same type as $C_{\infty}$ : or, the degeneration type of $C^{*}$ is equal to the dual deceneration type of $C$. For cxample, consider the degeneration type $\lambda: A$ is a (general) point, 3 a (general) plane. When $C$ degenerates along the cone over it, with vertex $A$, onto the plane $F$, its osculating planes degenerate towards the plane $B$. In $\breve{P}^{3}$, this means that $C_{a}^{*}$ degenerates on the cone with vertex the plane ${ }_{A}=\ddot{\mathbb{F}}^{3}$ towards the point $\breve{B} \in \mathrm{P}^{3}$. This degenoration type we call $\lambda^{\prime}$; in general, we shall denote the dual degeneration by a "prime" in this way.

\section{Scbubert's 11 derenerations}

We now rive a list of Schubert's 17 tynes of desenerations, in his order and usine his naves for then. $\lambda \quad A=$ general point (not or $C$, not on any tangent)

$B=$ seneral plane (not osculating, not containing any tangerti)

Take $A=(0,1,0,1), B: x_{3}+x_{1}=0$,

and new coorinates:

$$
x_{0}=x_{2}+x_{0}, x_{7}=x_{3}+x_{1}, x_{2}=x_{2}-x_{0}, x_{3}=x_{3}-x_{1} .
$$

Then $\mathrm{C}_{\mathrm{a}} \mathrm{a} \neq \mathrm{O}$, is given by

$$
\begin{aligned}
& X_{0}^{\prime}=u v^{2}+u^{3}, x_{1}^{p}=a v^{3}+a u^{2} v, x_{2}^{p}=u v^{2}-u^{3}, x_{3}=v^{3}-u^{2} v \\
& I_{a}=\left(a^{2}\left(X_{0}+x_{2}\right)\left(X_{0}^{q}+X_{2}^{p}\right)=\left(X_{1}-a_{1}\right)^{2}\right. \text {, } \\
& x_{1}^{2}-a^{2} x^{2}-a^{2}\left(X_{0}+x_{z}\right)^{2},-X_{1} X_{2}+2 x_{0} x_{3} \\
& \left.\left(x_{0}+x_{2}\right)\left(x_{1}+x_{3}\right)^{2}-a_{2}{ }^{2}\left(x_{0}+x_{2}\right)^{3}\right)
\end{aligned}
$$




$$
\begin{aligned}
& I_{0}=\left(X_{1}^{2}, X_{1} X_{3}, x_{j} x_{p}, X_{3}^{2}\left(X_{0}-X_{2}^{\prime}\right)-X_{2}^{2}\left(X_{0}^{p}+X_{2}^{p}\right)\right) \\
& =\left(\left(X_{3}+X_{1}\right)^{2},\left(X_{3}+X_{1}\right)\left(X_{3}-X_{1}\right),\left(X_{3}+X_{1}\right)\left(X_{2}-X_{0}\right)\right. \text {, } \\
& \left.X_{0}\left(X_{3}-X_{1}\right)^{2}-X_{2}\left(X_{2}-X_{0}\right)^{2}\right)
\end{aligned}
$$

Ilence: $C_{0}$ is a plane nodal cubic with a nomplanar embedded point at the node.

$\lambda^{\ominus} \quad A=$ Eeneral plane

$B=$ ceneral point

Take $A: X_{3}+X_{1}=0, B=(0,7,0,7)$ and coordinates as for $\lambda$.

$$
\begin{aligned}
& I_{a}=\left(\left(X_{0}-X_{2}\right)\left(x_{0}^{p}+X_{2}^{p}\right)-\left(a X_{1}^{p}-X_{3}^{p}\right)^{2}, a^{2} X_{1}^{2}-X_{3}^{2}-\left(X_{0}^{1}+x_{2}^{1}\right)^{2},\right. \\
& x_{0} x_{3}=2 x^{\prime}\left(x_{2}\right)
\end{aligned}
$$

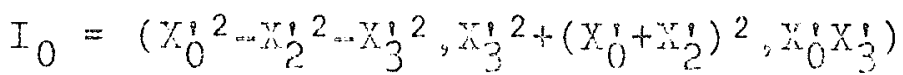

$$
\begin{aligned}
& =\left(4 x_{0} X_{2}-\left(X_{3}-X_{1}\right)^{2}, X_{2}\left(X_{2}+X_{0}\right),\left(X_{3}-X_{1}\right)\left(X_{2}+X_{0}\right)\right)
\end{aligned}
$$

Ilence: $C_{0}$ is the unjon of three skew lines through the point $(0,7,0,7)$.

To find the degenerated tangent curve ro of $\lambda$ (or of $\left.\lambda^{\prime}\right)$ :

$r_{a}$ is given (in coordinates $Y_{0}^{4}, \ldots, \frac{1}{5}$ on $\mathbb{P}^{5}$ corres ponding to $x_{0}^{3}, \ldots, x_{3}^{2}$ on $p^{3}$ ) on paraneter fom

$$
\begin{aligned}
& Y_{O}^{q}=v^{4}-2 u^{2} v^{2}+u^{4}, Y_{j}^{p}=4 a u v^{3}, Y_{2}^{p}=-a v^{4}+4 a u^{2} v^{2}+a u^{4} \text {, } \\
& y_{3}^{5}=v^{4}+4 u^{2} v^{2} \cdots u^{4}, \quad y_{1}=4 u^{3} v, \quad v^{4}=a v^{4}+2 a u^{2} v^{2}+a u^{4} \text {. }
\end{aligned}
$$

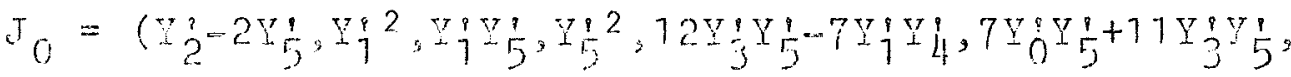

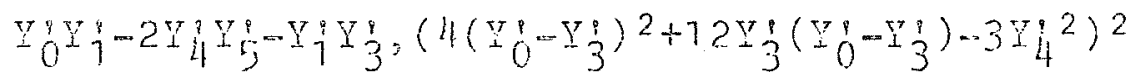

$$
\begin{aligned}
& \left.-\left(5 Y+4+4 \frac{1}{3}\right)^{2}\left(\left(Y_{0}^{2}-\frac{Y}{3}\right)^{2}+3 Y_{4}^{2}\right)\right) \text {. }
\end{aligned}
$$

Ience: $\Gamma_{0}$ is a plane tricuspidal quartic, with embeded points at the cusps.

$k \quad A=$ point on a tangent, not on $C$

$B=$ plane containing a tangent, not osculating.

Talie $A=(0,1,0,0), B: I_{1}=0$. Then $C_{a}$ a 0 , is given by

$$
x_{0}=u^{3}, x_{1}=a u^{2} v_{2} x_{2}=u v^{2}, x_{3}=v^{3},
$$




$$
\begin{aligned}
& I_{a}=\left(a^{2} X_{0} X_{2}-X_{1}^{2}, X_{1}, X_{3}-\mathrm{aX}_{2}^{2}, a X_{0} x_{3}-X_{1} X_{2}, X_{2} X_{3}^{2}-X_{2}^{3}\right) . \\
& I_{0}=\left(X_{1}^{2}, X_{1} X_{2}, X_{1} X_{3}, X_{0} X_{3}^{2} x_{2} X_{2}^{3}\right) \text { 。 }
\end{aligned}
$$

Hence: $C_{0}$ is a cuspidal cubic, in the plane $x_{1}=0$, with a nonplanar embedied point at the cusp.

$K^{\prime} \quad A=$ plane containing a tangent, not osculating.

$B$ = point on tarigent, not on $\mathrm{C}$.

$I_{a}=\left(x_{0} x_{2}-a^{2} x_{1}^{2}, a X_{1} x_{3}-x_{2}^{2}, x_{0} x_{3}-a X_{1} x_{2}\right)$

$I_{0}=\left(X_{0} X_{2}, X_{2}^{2}, X_{0} X_{3}\right)$

Hence: $C_{0}$ is the union of the line $x_{2}=x_{3}=0$ with the double line $X_{0}=x_{2}=0$ (doubled on a quadratic cone with vertex $(0,1,0,0))$.

The tangent curve $\Gamma_{a}$ of $k\left(\right.$ or $\left.k^{8}\right)$ is given by

$$
\begin{aligned}
& Y_{0}=v^{4}, Y_{1}=2 a u v^{3}, Y_{2}=a^{2} v^{2}, \\
& Y_{3}=3 u^{2} v^{2}, Y_{4}=2 u^{3} v_{3} Y_{5}=a v^{4} . \\
& J_{0}=\left(Y_{2}, Y_{7}, Y_{7} Y_{3}, Y_{7} Y_{4}, Y_{7} Y_{5}, Y_{0} Y_{5}, Y_{2}{ }^{Y_{5}}, 27 Y_{0} Y_{4}^{2}-4 Y_{3}^{3}\right) .
\end{aligned}
$$

Hence: $T_{C}$ is a cuspidal cubic, in the plane $Y_{1}=Y_{2}=Y_{5}=0$, with a nonplanar eribedded point at the cusp $(1,0,0,0,0,0)$ (this point corresponds to the flex tangent of $\left.C_{0}\right)$, union the line $Y_{0}=\stackrel{y}{+}_{1}=Y_{2}=Y_{3}=0$, intersecting the cubic in its flex $(0,0,0,0,1,0)$ (corresponding to the cusp tangent of $\mathrm{C}_{0}$ ).

$\omega \quad A=$ point on $C$

$\mathrm{B}=$ osculating plane

Thake $A=(0,0,0,1), \quad 33: Y_{3}=0$

$\mathrm{C}_{a}$ a $\mathrm{a}$, is given by

$x_{0}=u^{3}, x_{1}=u^{2} v, x_{2}=u v^{2}, x_{3}=a v^{3}$,

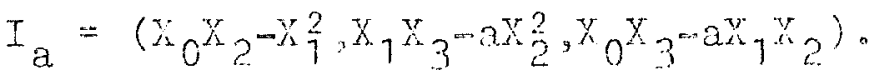

$I_{0}=\left(x_{0} x_{2} x_{1}^{2}, x_{0} X_{3}, x_{1}^{x_{3}}\right)$

Hence: $C_{0}$ is the union of a conic, in the plane $x_{3}=0$, with the line $x_{0}=x_{1}=0$ 。 
$\omega^{\prime} \quad A=$ osculating plane

$B=$ point on $C$

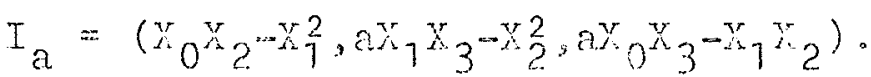

$I_{C}=\left(X_{0} X_{2}-X_{1}^{2}, X_{1} Y_{2}, X_{2}^{2}\right)$

Hence: $C_{0}$ is the triple line $y_{1}=x_{2}=0$ (tripled on a quadratic cone with verter $(0,0,0,1))$.

The tansent curve $\Gamma_{\text {a }}$ of $(\omega)\left(\right.$ or $\left.\omega^{\prime}\right)$ is miver by

$$
\begin{aligned}
& Y_{0}=a v^{4}, Y_{1}=2 a u v^{3}, Y_{2}: u^{2} v^{2}, Y_{3}=3 a u^{2} v^{2}, \\
& Y_{4}=2 u{ }^{3} v, Y_{5}=u u^{4} . \\
& J_{0}=\left(Y_{3}, Y_{7}^{2}, Y_{1} Y_{4}, Y_{7} Y_{5}, Y_{0} Y_{5}, Y_{0} Y_{4}-Y_{1} Y_{2}, Y_{2} Y_{5}-Y_{4}^{2}\right) .
\end{aligned}
$$

Hence: $\Gamma_{0}$ is the union of a conic, in the plare $Y_{0}=Y_{1}=Y_{3}=0$, with the dounle line $y_{1}=I_{3}=y_{14}=y_{5}=0$ 。

$\theta$ To obtain this degeneration, we choose $\Lambda$ to be a "Iinewplane" $(I, U)$, sot. for sone $x \in C, x \in I, C U$, $t_{x} \subset \cdot, L * t_{x}$, U not osculating - and $B$ a. "point-line" $(P, I ")$, s.t. ror sone $x \in C, L^{\prime} \in$ osc $_{x}$, $\{P\}=L^{*} \cdot t E_{X}, x \neq P, P \neq C$. Then we form a

2 dimensional family $\{\mathrm{C}, \mathrm{a}, \mathrm{b}\}$, where the parameter a corresponds to projecting $C$ from $U$ to $P$, and $b$ to projecting from It to $I_{4}$. Taking $a=b$ we obtain a 1-ainensional ramily $\left\{\mathrm{C}_{\mathrm{a}, \mathrm{a}}\right\}$.

Make $L: X_{1} x_{3}=x_{2}=0, U: X_{2}=0$ and $L^{\prime}: x_{0}=X_{1}+x_{3}=0$, $\mathrm{P}=(0,0,1,0)$. In new coorainates $X_{0}, X_{1}^{p}=X_{1}{ }^{-X_{3}}{ }^{s}$ $x_{2}, x_{3}=x_{1}+x_{3}, c_{a}, b$ js given by $x_{0}=a b u^{3}$, $x_{1}^{8}=a u^{2} y-a v^{3}, x_{z}=u v^{2}, x_{3}=a b u^{2} v+a b v^{3}$

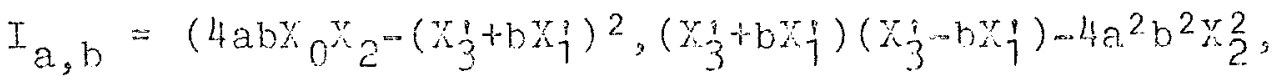

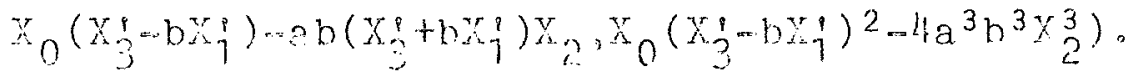

By letting $a=b$, rewritins the cenorators, and letting $a=0$, ve obtain $I_{0}=\left(X_{0}\left(X_{1}+X_{3}\right),\left(X_{1}-x_{3}\right)\left(X_{1}+x_{3}\right),\left(X_{1}+X_{3}\right)^{2}, x_{0}\left(\left(X_{1}-x_{3}\right)^{2}-x_{0} x_{2}\right)\right)$. 
Hence: $C_{0}$ is the union of a conic, in the plane $X_{1}+X_{3}=0$, with its tangent line at $(0,0,1,0)$, and with that point as a nonplanar erbedded point.

$$
\begin{aligned}
& \theta^{\prime} \quad A=\text { "point-Iine" } \\
& B=\text { Iine-plane". } \\
& I_{a, b}=\left(4 b x_{0} x_{2}-a\left(b x_{3}+x_{j}\right)^{2}, a^{2}\left(b X_{3}+x_{j}\right)\left(b X_{3}^{i}-X_{j}\right)\right. \\
& \left.-4 x_{2}^{2}, a b x_{0}\left(b x_{3}-x_{j}\right)-\left(b x_{3}+x_{1}^{p}\right) x_{2}\right) \text { 。 }
\end{aligned}
$$

Waling $a=b$ and $a=0$ eives

$$
I_{0}=\left(4 X_{0} x_{2}-\left(X_{1}-X_{3}\right)^{2},\left(X_{1}-X_{3}\right) X_{2}, X_{2}^{2}\right)
$$

Hence: $C_{0}$ is the line $X_{1} \cdots_{3}=X_{2}=0$ tripled on a quadratic cone with vertex $(1,0,1,0)$.

The tangent curve $\Gamma_{a}$ of $\theta\left(o r A^{\circ}\right)$ (for a $=b$ ) is given by

$$
\begin{aligned}
& Y_{O}^{\prime}=a v^{4}-a u^{2} v^{2}, Y_{j}^{\prime}=4 a^{2} u v^{3}, Y_{2}^{g}=v^{4}+u^{2} v^{2} \\
& Y_{3}^{8}=3 a^{3} u^{2} v^{2}+a^{3} u^{4}, Y_{4}=2 a u^{3} v, Y_{5}^{9}=-3 a^{2} u^{2} v^{2}+a^{2} u^{4} .
\end{aligned}
$$

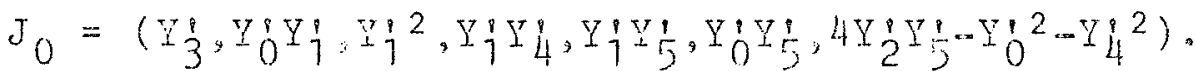

Hence: $\Gamma_{0}$ is the union of a conje, in the plane

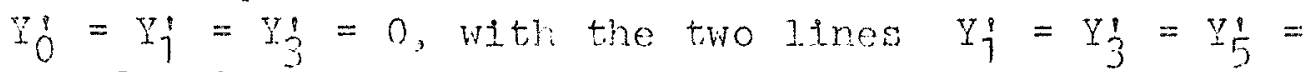
$=\mathrm{Y}_{0}^{2}+\mathrm{I}^{2}=0$, with the comon point of in erscetion, $(0,0,1,0,0,0)$, as an embedded point (this point corresponds to the line - tangent to the conic - of $\mathrm{C}_{0}$ ).

$\delta \quad \Lambda=$ a line, not contained in any osculatins plane, and intersecting, $C$ in exactly one point.

$$
\begin{aligned}
\Gamma= & \text { a line, not intersecting } C, \text { contained in } \\
& \text { exactly one osculating plane. }
\end{aligned}
$$

Talse $A:{ }^{X_{0}}{ }^{x_{2}}=x_{2}=0, P: X_{0}+x_{2}=y_{3}=0$ and change coordinates: $\quad x_{0}^{x_{0}}=x_{0}-X_{2}, x_{1}, x_{2}=X_{0}+x_{2}, x_{3}$, ihen $c_{a}$ is given by

$$
\begin{aligned}
& { }^{x} y=u^{3}-u v^{2}, x_{1}=u^{2} v, x_{2}=a u^{3}+a u v^{2}, x_{3}=a v^{3} .
\end{aligned}
$$

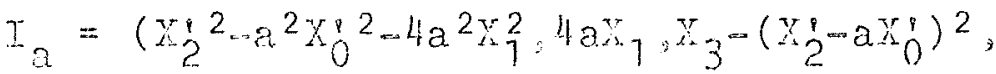

$$
\begin{aligned}
& \left.\left(x_{2}+a x_{0}^{8}\right) x_{3}-a x_{1}\left(x_{2}^{p}-a x_{0}^{p}\right)\right)
\end{aligned}
$$


By changing the generators, we see

$$
I_{0}=\left(\left(X_{0}+X_{2}\right)^{2},\left(X_{0}+X_{2}\right) X_{3}, X_{0}^{2}-X_{2}^{2}-2 X_{7} X_{3}\right)
$$

Ilence: $C_{0}$ is the union of the line ${ }_{x_{0}}+x_{2}={ }_{21}^{r r}=0$ with the double line $\mathrm{x}_{0}+\mathrm{x}_{2}={ }_{x_{3}}=0_{4}$ and is contained in a smooth quadric.

$$
\begin{aligned}
& \delta^{\prime} \quad I_{a}=\left(a^{2} X_{2}^{2}-y_{0}^{2}-4 X_{1}^{2}, 4 a X_{1} X_{3}-\left(a X_{2}-X_{0}\right)^{2},\right.
\end{aligned}
$$

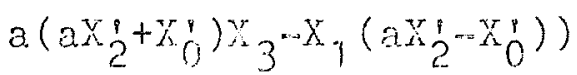

$$
\begin{aligned}
& I_{0}=\left(\left(X_{0}-X_{2}\right)^{2},\left(X_{0}-X_{2}\right) X_{1}, X_{1}^{2}\right)=\left(X_{0}-X_{2}, X_{1}\right)^{2}
\end{aligned}
$$

Hence: $C_{0}$ is the triple line ${ }^{T_{0}}{ }_{0}-\mathrm{K}_{2}=\mathrm{X}_{1}=0$ (tripled by taking j.ts and order neighbourhood in $\mathbb{P}^{3}$ ).

me tangent curve $\Gamma_{a}$ for $\delta\left(\right.$ or $\delta^{\prime}$ ) is given by

$$
\begin{aligned}
& Y_{0}^{q}=a^{2} v^{4}+3 a^{2} u^{2} v^{2}, \quad Y_{1}^{p}=2 a u v^{3}, Y_{2}^{p}=a u^{2} v^{2}-a u^{4}, \\
& Y_{3}^{\prime}=-a v^{4}+3 a u^{2} v^{2}, Y_{H_{1}}=4 a u^{3} v, Y_{5}^{\prime}=u^{2} v^{2}+u^{4} . \\
& J_{0}=\left(Y_{0}^{q}, Y_{7}^{q}\left(2 Y_{j}+Y_{4}^{q}\right), Y_{2}\left(2 Y_{7}+Y_{4}\right), Y_{4}\left(2 Y_{7}^{q}+Y_{L}^{q}\right) .\right.
\end{aligned}
$$

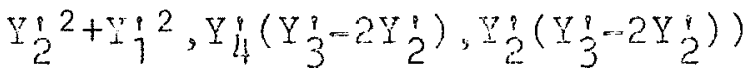

Hence: $I_{0}$ is the union of the two lines

$$
\begin{aligned}
& Y_{0}^{q}=2 Y_{1}^{q}+Y_{4}=Y_{3}^{8}-2 Y_{2}^{\prime}=Y_{1}^{2}+Y_{2}^{2}=0 \text {, wi.th the double } \\
& \text { line } Y_{0}^{\prime}=Y_{1}^{q}=Y_{2}^{q}=Y_{4}=0 .
\end{aligned}
$$

$\eta \quad A=$ general Iine, joe. $A \cap C=\not$, A not contained in an osculating plane

$$
B=\text { General Iine (same conditions as for } A \text {, }
$$
since these are self-aval!)

Take $A: X_{0}-x_{2}=x_{1}+x_{2}=0, B: x_{0}+x_{3}=x_{1}=x_{2}=0$, and change coordinates:

$x_{0}^{p}=x_{0}-x_{3}, x_{1}=x_{1}+x_{2}, x_{2}=x_{1}+y_{2}, x_{3}=x_{0}+x_{3}$. Then

$\mathrm{C}_{\text {a }}$ is given by

$$
\begin{aligned}
& x_{0}=u^{3}-v^{3}, x_{1}^{p}=a u^{2} v \cdots a u v^{2}, x_{2}=u^{2} v+u v^{2}, \\
& x_{3}=a u^{3}+a v^{3} .
\end{aligned}
$$




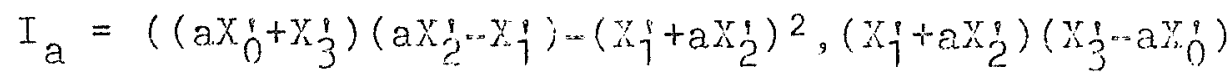

$$
\begin{aligned}
& \left.-\left(a X_{2}^{q}-X_{j}^{p}\right)^{2}, X_{3}^{p}-a^{2} X_{0}^{2}-a^{2} X_{2}^{2}+X_{j}^{2}\right) \\
& I_{0}=\left(X_{j}^{2}, X_{1} X_{3}^{1}, x_{3}^{2}\right)=\left(X_{1}, X_{2}, X_{0}+X_{3}\right)^{2}
\end{aligned}
$$

Fience: $C_{0}$ is the tripled line $x_{1} x_{2}=x_{0}+y_{3}=0$ (tripled as in $\varepsilon^{*}$ ).

$\eta^{\prime}$ is of the sane type as $\eta$, since the conditions on

$A, B$ are self dual.

The tangent curve $\Gamma_{a}$ of $n$ is iven by

$$
\begin{aligned}
& Y_{0}^{\prime}=a v^{4}+2 a u v^{3}-2 a u^{3} v-a u^{4} \\
& Y_{i}^{p}=-a^{2} v^{4}+2 a^{2} u v^{3}+2 a^{2} u^{3} v-a^{2} u^{4} \\
& Y_{2}=2 \mathrm{au}^{2} \mathrm{v}^{2} \\
& Y_{3}^{\prime}=6 a u^{2} v^{2} \\
& Y=v^{4}+2 u v^{3}+2 u^{3} v+u^{4} \\
& Y \frac{9}{5}=-a v^{4}+2 a u v^{3}-2 a u^{3} v+a u^{4} .
\end{aligned}
$$

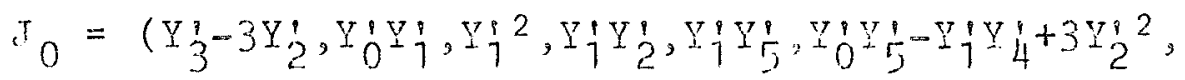

$$
\begin{aligned}
& 4 Y\left(Y Y_{4}^{8}-6 Y_{0}^{8} Y_{5}^{8}+3 Y_{0}^{2}-Y_{5}^{2}\right)
\end{aligned}
$$

Ience: $\Gamma_{0}$ is the union of four lines in the three-space $Y_{3}-3 Y_{2}^{\prime}=Y_{1}^{p}=0$, with an embedied point (sticleins out of that space) at their comon point of intersection.

Rerark: By choosins other A's and D's we can obtain further types of defenerations. For example, consider the degeneration obtained by taking $A=$ a chord of $C, B=$ an axis of $C$ (i.e., the intersection of two osculating planes). Then $C_{0}$ is the union of three skew lines, neeting in 2 points, wereas its dual. is a triple ljne (and order nbhd. of a line in $\left.\breve{F}^{3}\right)$. The tansent curve $\Gamma_{0}$ is the union of tro double lines.

On the next pare, we give a figure showing schubert's 11 degenerate complete twister cubics. Each trinle should also be read hackwards! 
$-11-$
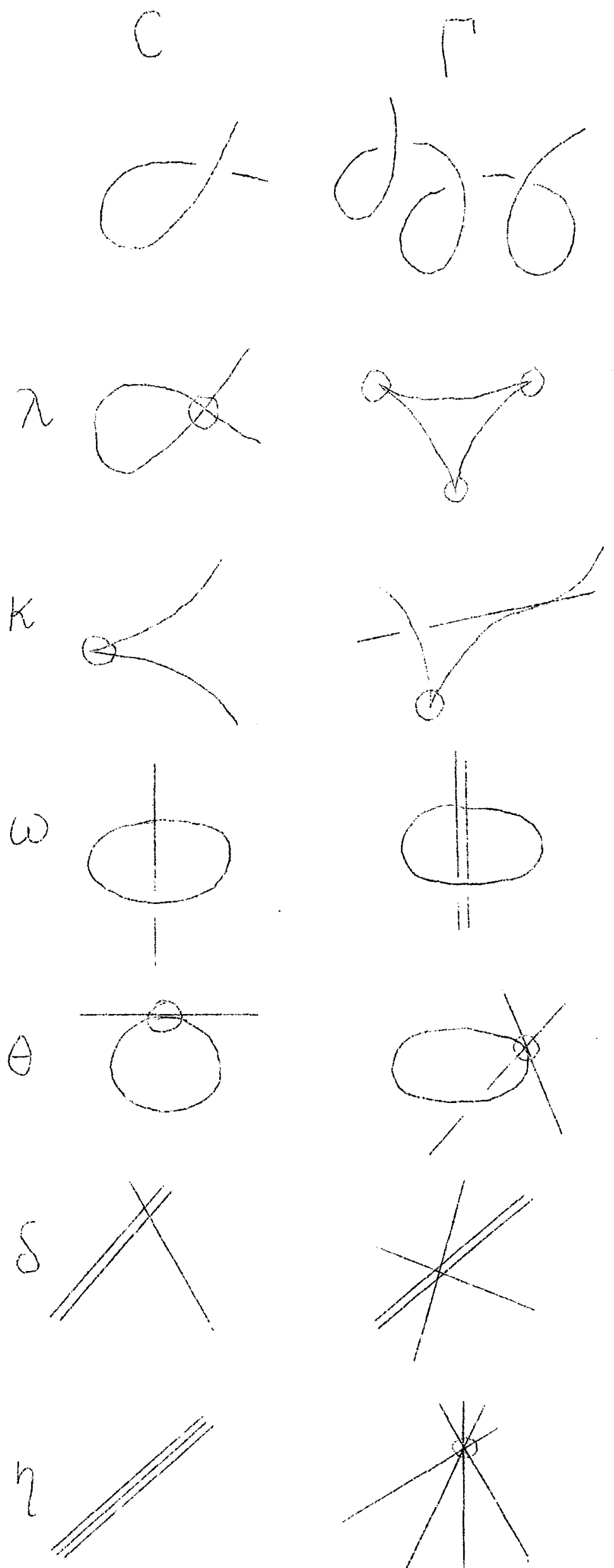
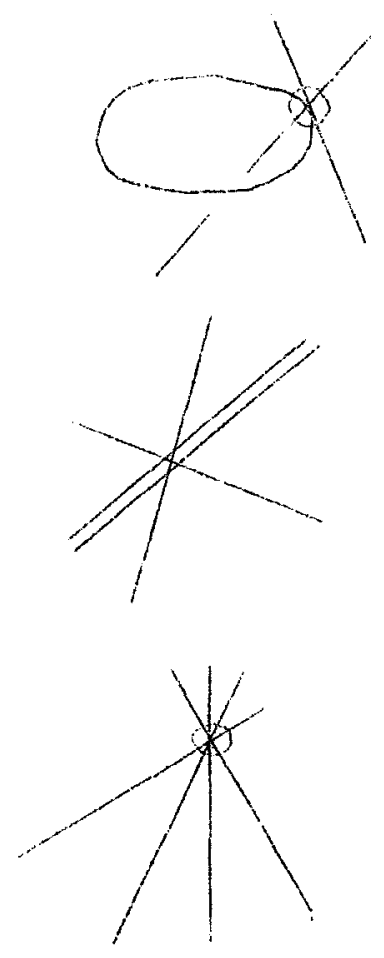
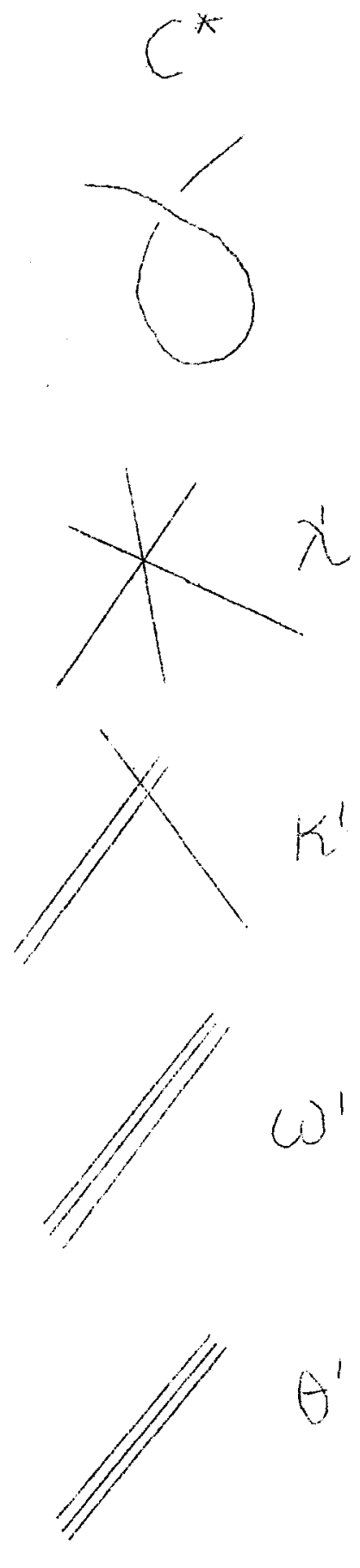

$\theta^{\prime}$

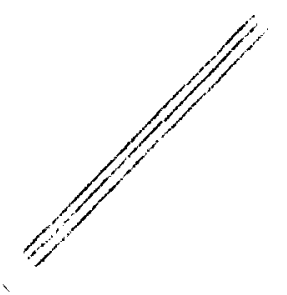

$\delta^{\prime}$

$\eta^{\prime}$ 
4. Some remarks on $H$ and $T$

Let $T_{\lambda}, T_{K} \ldots$ denote the closure of the set of points in $T$ corresponding to degenerations of type $\lambda, k, \ldots$, and let $I_{\lambda}, I_{k}, \ldots$ denote the sirilarly defined sets in ${ }_{1}$. That the degenerations $\lambda, k, \ldots$ are of first order, neans that ${ }^{\mathrm{r}} \lambda, \mathrm{T}_{K}, \ldots$ are of codinension 1 in $T$ : this is easily seen to be true by counting the paraneters of each of the corresponding firures, only $I_{\lambda}$ and $I_{u}$ are of codjriension 1 in $\mathrm{H}$, so the (birational) projection map $\pi: \Gamma \rightarrow I$ blows up the other sets ${ }_{K}{ }{ } \lambda_{\lambda}, \ldots$. For example, $H_{k}$ has codimension 2 (there are ${ }^{10}$ plane cuspidal cubics in $\mathrm{p}^{3}$ ), and for a given C $H_{K}, \pi^{-1}(\bar{C})=$

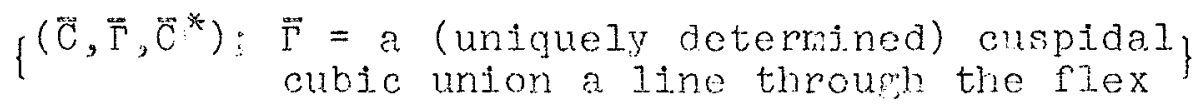
Since "a. line through the flex" corrosponds to "a plane containing the cusp tansent of $\vec{C} "$, we see that dirn $\pi^{\infty+1}(\tilde{C})=1$.

The set $\|_{n}\left(={ }^{\prime}{ },\right)$ has the larsest codimension, namely 8 ; all derenerations without an embedded point specialize to these. In this case, $\pi^{-1}(\mathrm{C})$ has dimension 7: the tanpent curve js detemined by choosing 4 pointplanes through the line Cred, which satisfy one relation between the cross-ratios (of the points and planes) (see e.E. [A], p.206, or recal] that the four concurrent lines $\tilde{\Gamma}_{\text {red }}$ span only a $p^{3}$ ).

Let it denote the nomal sheaf of $\bar{C} \in \|_{n}$ in $\mathbb{P}^{3}$. One can prove e.s. by taking a presentation of the ideal of $\bar{C}$, that $\operatorname{din} I^{0}(N, \bar{C})=12$. It follows that $I$ is snooth at $\bar{C}$, since dirn $11=12$, and hence all points of II-IA (i.f., those corrosponting to Cohen-racaulay curves, i.e., curves without an emberded point) are smooth on Ho Now consider It $\lambda$. Any point in it can be specialized to one correspondins, to a plane triple line with a nonplanar erobedied point, e.g. given by the ideal $\left(x_{1} x_{3}, x_{2} x_{3}, x_{3}^{2}, x_{1}^{3}\right)$. In the work with $r$. Schlessinger, cited in the introduction, we nrove that such a point is smooth on II, and hence thot If is smooth. 
Remark: The results $\operatorname{inn} \mathrm{H}^{0}(\mathrm{H}, \overline{\mathrm{C}})=12$ if $\overline{\mathrm{C}} \in \mathrm{H}_{\eta}$, and $\operatorname{din} \mathrm{H}^{0}(\mathrm{~N}, \overrightarrow{\mathrm{C}})=16$ if $\overline{\mathrm{C}}$ is a plane triple line with embedded point, have also been obtained by Joe Harris; he also gives a list of possible degeneration types of a curve $\mathrm{C} E \mathrm{H}$ (private comunication).

As a final comment, let us rention an advantage of working with Hilbert schemes rather than Chow schemes: the existence of universal families of curves, which allows the following way of expressing Schuhert's various conditions as cycles on m. Namely, let

\begin{tabular}{|c|c|c|}
\hline$C \subset \mathbb{P}^{3} \times T$ & $e^{\prime} C \operatorname{Crass}(1,3) \times T$ & $e^{*}<\check{p}^{3} \times m$ \\
\hline$\downarrow$ & $\downarrow$ & $r \downarrow$ \\
\hline
\end{tabular}

denote the universal families (pulled back to From II. c, II respectively). The condition, denoted $v$ by Schubert, for a curve $C$ to intersect a given line $I_{3}$ is then represented by the cycle $w_{\nu}=p_{*}(C \cap I \times M)$; the condition, Schubert's $\rho$, that the curve touches a diven plane $U$, by $T_{\rho}=a_{*}\left(u^{\prime} n \sigma_{1,1} \times \mathrm{T}\right)$, where $\sigma_{1,1}$ is the 2-plane in Grass $(1,3)$ of lines in $U$, and so on. We plan to return to the question of detemining the relations between these cycles and the cycles ${ }_{{ }_{1}}^{T_{1}} \lambda T_{K}, \ldots .$. and to a study of the choy ring of $\mathrm{r}$.

\section{Bibliograngy}

[A] A.R. Alguneid, "Analytical didegeneration of comlete tvisted cubics" Proc. Cambridge Phil.Soc. 52(1956); $202-208$.

[H] Ho Ilartshorne; Almebraic Coonetry. New Zork-Ifeidelherg - Derlin, Springeroverlas 7977.

[P] R. Piene, "Tumerical characters of a curve in projective n-space". In Real and compley sinvularities, Oslo 1976 . Ed. P. Iolno Croningen: Sijthofi and loordhoff $7978 ;$ pp. $175-495$.

[S] H. Schubert, Iralkül der abzählenden ceometrie. B.r. Toubner, Leiprig 7879. (New edition: Springerm Verlag, 197\%) 